\title{
How is difficult asthma managed?
}

\author{
N.J. Roberts*, D.S. Robinson* and M.R. Partridge*
}

ABSTRACT: Most patients with asthma can be easily treated. Some have difficult asthma; in some because the diagnosis is erroneous, in others because of comorbidity or noncompliance. A European Respiratory Society Task Force has called for an integrated approach for these patients, and positive results have been reported using protocols. In the UK, there is no overall understanding of the size of this problem, or how these patients are managed.

A postal survey of 683 consultant members of the British Thoracic Society designed to elicit respondents' views on how they would manage four clinical scenarios was conducted.

There was a $\mathbf{5 0 . 4 \%}$ response rate. Few reported a uniform approach to the investigation of such patients. The availability of allied healthcare professionals was variable. The 21 consultant respiratory physicans, reporting a special interest in difficult asthma, were significantly more likely to objectively assess compliance, perform skin-prick tests and to utilise a liaison psychiatrist than those without an expressed special interest in asthma. Many reported difficulty in accessing psychologists, liaison psychiatrists and social workers. Approaches to the diagnosis and management of "vocal cord dysfunction" were variable.

The results of this postal survey of specialist thoracic physicians in the UK suggest that a protocol for difficult asthma is not in widespread use and that access to necessary allied healthcare professionals is not uniform. Pulmonologists with a declared special interest in difficult asthma may have configured their services and approaches more in line with that proposed by the European Respiratory Society Task Force.

\section{KEYWORDS: Delivery of care, difficult asthma, liaison psychiatry}

n 1998, a European Respiratory Society (ERS)

Task Force on difficult/therapy-resistant asthma called for an integrated approach to define clinical phenotypes, evaluate risk factors, understand pathophysiology and find novel therapies [1]. Subsequently, the European Network for Understanding Mechanisms of Severe Asthma (ENFUMOSA) study group [2] have published details of 163 subjects with severe asthma, who have been carefully studied, and compared those with 158 milder-controlled patients with asthma; the Outcomes and Treatment Regimens (TENOR) study shows similar data from the USA [3]. The ERS Task Force specifically recommended the use of a protocol for evaluating patients with difficult asthma, which suggested a number of assessments and investigations that should be undertaken for these patients, including, for example, psychological assessment. Similarly, a protocol approach had been recommended from the USA as long ago as 1993 [4]. Subsequently, results from single centres of the value of a systematic assessment of these patients have shown the advantages of such an approach in detecting

For editorial comments see page 897 . misdiagnosis, significant major psychiatric comorbidity and high rates of nonadherence with oral steroid therapy [5]. Others have subsequently shown that such an approach reveals that approximately only half $(46.5 \%)$ of cases had truly therapy-resistant asthma [6].

Most national and international guidelines on the management of asthma do not address the issue of difficult asthma, although one group has recently published specific recommendations [7]. The size of the problem is not known, but the burden for the individual and healthcare system is likely to be considerable. Many patients requiring unscheduled healthcare do so in a repetitive manner [8]. Resource utilisation is approximately three times higher in patients with poorly controlled severe asthma, compared with well-controlled, persistent asthma [9] and these patients incur significant indirect costs due to time off work [10].

Within the group of people with difficult asthma, there will be some in whom there is significant comorbidity, some in whom the diagnosis is erroneous, some who have significant psychological problems and some who fail to benefit from therapy as a result of noncompliance.
AFFILIATIONS

${ }^{*}$ Respiratory Health Services Research Group, Charing Cross Hospital, and

\#Leukocyte Biology Section and Dept of Allergy and Clinical Immunology, NHLI Division, Imperial College London, London, UK.

CORRESPONDENCE

M.R. Partridge

Respiratory Health Services Research Group

NHLI Division

Imperial College London

Charing Cross Hospital

St Dunstans Road

London

W6 8RP

UK

Fax: 442088467999

E-mail: m.partridge@imperial.ac.uk

Received:

January 112006

Accepted after revision:

June 162006
European Respiratory Journal Print ISSN 0903-1936 Online ISSN 1399-3003 


\section{METHODS}

A postal questionnaire survey was sent out to 683 consultant members of the British Thoracic Society. Members known to be paediatricians, radiologists or thoracic surgeons were excluded. A covering letter stressed the anonymous nature of the survey and respondents were asked to complete the form themselves rather than pass it to a colleague. If received inadvertently by a paediatrician or by someone not in clinical practice, they were asked to notify us and return the form uncompleted.

The questionnaire consisted of five parts; the first four parts concerned case histories of difficult asthma and a fifth section was designed to elicit information about the respondent's place of work.

The case studies were based upon cases seen previously by the authors, but altered and made anonymous. Case 1 concerned a patient with multiple admissions and episodes of mechanical ventilation who was on maximal therapy. Respondents were asked to tick a list of investigations they would perform on the majority of patients of this type. Case 2 was designed to elicit the pattern of services available to patients with difficult asthma and concerned a student with asthma that had recently worsened despite maximal therapies. The respondent was asked to record which other health professionals were physically present in their clinic at the time of referral and who was easily available by onward referral within 2 or 3 weeks. Case 3 was designed to elicit a list of differential diagnoses and concerned a young male with asthma since early childhood, who had never been fully investigated because of severe anxiety and needle phobia. Respondents were asked to list five possible alternative diagnoses and also to list the three most common misdiagnoses they had seen in patients attending with asthma. They were also asked to list the most unusual diagnosis they have ever made in somebody originally labelled as having difficult asthma. Case 4 concerned a young female with asthma who had had frequent emergency department attendances, and was noted to have extremely variable spirometry and a glottic wheeze. Respondents were asked to state the likely diagnosis and to list which investigations, if any, they used to confirm the diagnosis and to describe who they would most involve in therapy in such a case.

The fifth section elicited information about the clinician completing the questionnaire, including their place of work, whether there was an asthma clinic or difficult asthma clinic in their hospital and whether they were the clinical lead for that condition. They were also asked how many patients with difficult asthma they currently cared for.

\section{Statistical analysis}

Variables were treated non-parametrically and analysed using the Mann-Whitney U-test.

\section{RESULTS}

A total of 344 questionnaires were returned, a 50.4\% response rate, of which 310 were completed and 34 were returned incomplete; four because the recipient had retired, 11 because the content was not perceived to be relevant to their speciality, five stated they were no longer in clinical practice, seven did not see patients with asthma, one was from a locum who did not feel it appropriate to complete the form, and six were returned because the recipient was unknown at that address. Some forms were incomplete because for each of the case histories the respondent could report that they would not usually see this sort of case or that they would refer them to colleagues.

The investigations reported to be undertaken on those with difficult asthma are shown in figure 1 and the availability of health professional colleagues is shown in figure 2 . The five most common differential diagnoses offered for case 3 are shown in figure 3 and the complete list of misdiagnoses reported by respondents to have mimicked difficult asthma is illustrated in table 1.

In total, 233 respondents thought that the likely diagnosis for the clinical scenario outlined in case 4 was that the patient had "upper airway obstruction", "vocal cord dysfunction", "glottic wheezing" or similar words, 33 reported the problem to be psychological and 22 diagnosed hyperventilation syndrome or dysfunctional breathing. A wide array of investigations were reported to be used in such cases: 192 reported that they used flow-volume curves and spirometry; 113 requested an ear, nose and throat opinion; 102 undertook a bronchoscopy and bronchoscopic examination of the vocal cords; 47 reported measuring bronchial hyperresponsiveness; 41 serial peak flow recording; 15 blood gas estimation; and 13 computed tomography scanning. There was a similarly diverse response to the question as to which healthcare professional respondents were involved most in the therapy for these patients, the most common of whom are listed in figure 4 .

\section{Demographics of respondents}

Of the 293 respondents, 266 (90.8\%) were consultant respiratory physicians with the remainder being university senior lecturers, readers, professors or staff grade physicians. In total, 140 worked at a district general hospital, 94 in a teaching hospital, 42 in an associated university hospital and 18 in a regional cardiothoracic centre.

\section{Numbers of patients with difficult asthma}

A total of 280 respondents reported currently caring for a mean of 25.1 patients. The total number of patients under the care of the 280 consultants is 7,027 , of whom approximately half $(3,635)$ were under the care of the 21 consultants who declared that they had a special interest in difficult asthma.

\section{Specific clinics for those with asthma or difficult asthma}

Forty-eight per cent (140 out of 292) respondents said that in their hospital one consultant was lead on asthma, $55(39.3 \%)$ of whom were the lead on asthma themselves. Out of 293, 122 $(41.6 \%)$ had a specific clinic in their hospital mainly for people with asthma.

Only 65 out of $287(22.7 \%)$ had a specific clinic for those with difficult asthma in their hospital. Of 284 respondents, 183 $(64.4 \%)$ answered that there was someone in their regional centre with an interest in difficult asthma and 21 of those 183 $(11.4 \%)$ were themselves the specialist for difficult asthma.

The responses of the 21 respiratory specialists (table 2), who reported having a special interest in difficult asthma, were compared with the 152 doctors who reported neither a 


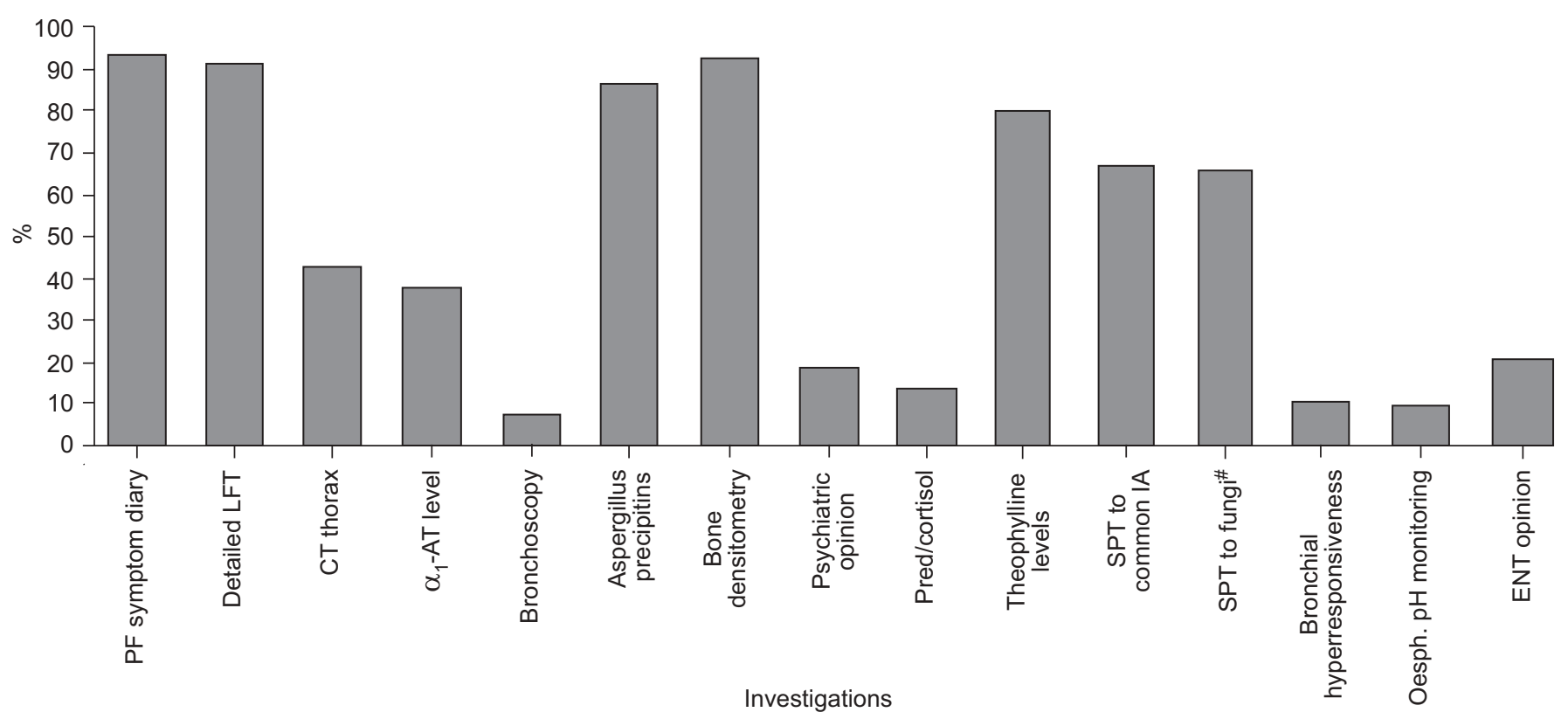

FIGURE 1. Investigations frequently performed on the majority of patients with difficult asthma (case 1). Data are presented as percentage of patients investigated. PF: peak flow; LFT: lung function testing; CT: computed tomography; AT: antitrypsin; Pred: prednisolone assay; SPT: skin-prick test; IA: inhaled allergens; Oesph.: oesophageal; ENT: ear, nose and throat. \#: i.e. aspergillus, cladosporium.

specialist responsibility for asthma or for difficult asthma. Responses from the former showed a significantly higher use of liaison psychiatric opinion ( $\mathrm{p}=0.002)$, prednisolone assays / cortisols $(p=0.028)$ and use of skin-prick tests for common inhaled allergens and fungi $(\mathrm{p}=0.012$ and $\mathrm{p}<0.001$ respectively). The most significant results are the increased ease of access from a difficult asthma clinic to a liaison psychiatrist $(p=0.001)$. The 21 difficult asthma specialists also had a significantly increased number of patients with difficult asthma $173.09 \pm 232.7$ (mean \pm SD) compared to the mean $10.43 \pm 13.60$ cared for by the remaining respiratory physicians.

\section{DISCUSSION}

Seven years after an ERS Task Force recommended an integrated approach to the problem of difficult asthma [1] and 13 years after the value of a systematic approach was demonstrated to be helpful [5], within one European country at least, a systematic approach is not the approach of most

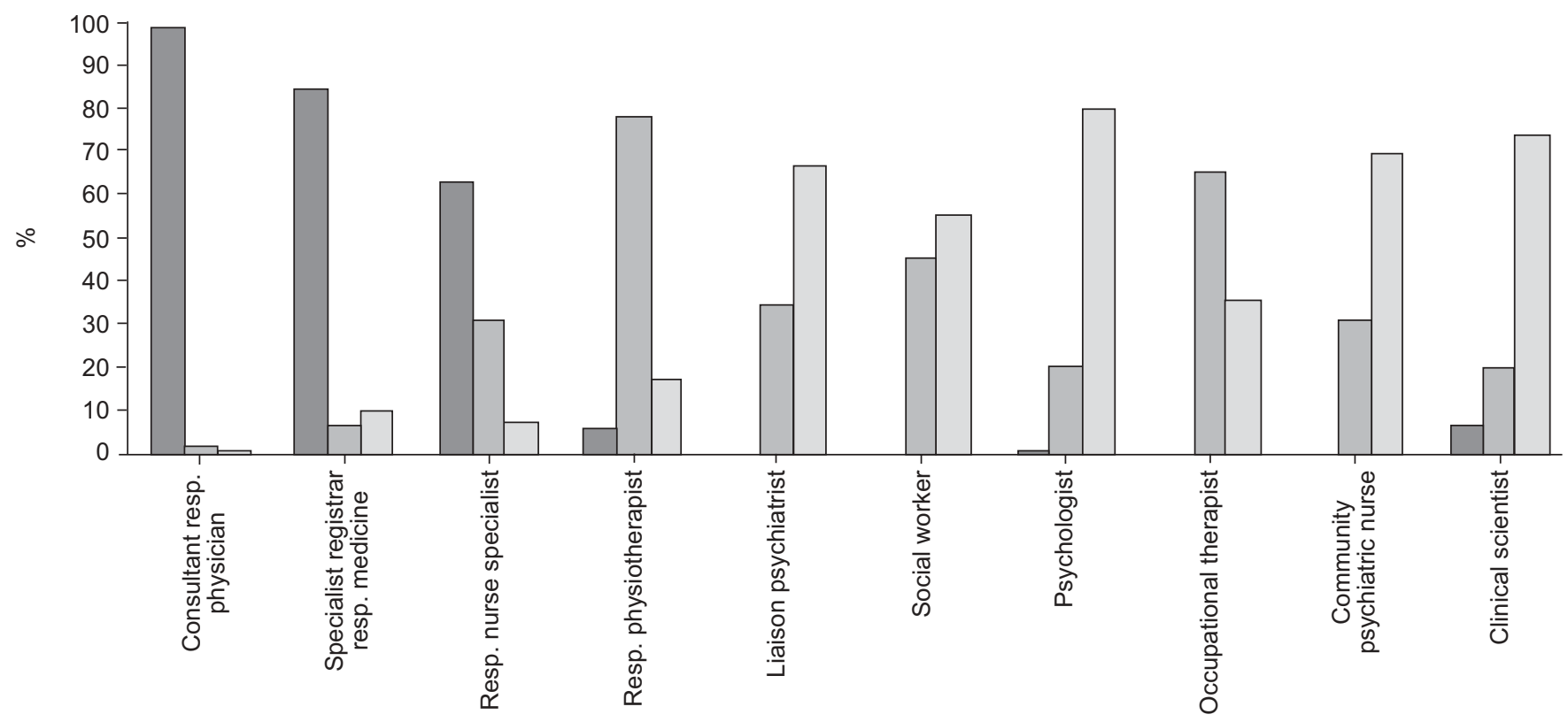

FIGURE 2. Percentage of doctors reporting the availability of other healthcare professionals in their outpatient clinics. Resp.: respiratory. $\square$ : physically present in clinic; 口: easily available by referral; 1 : difficult to access. 


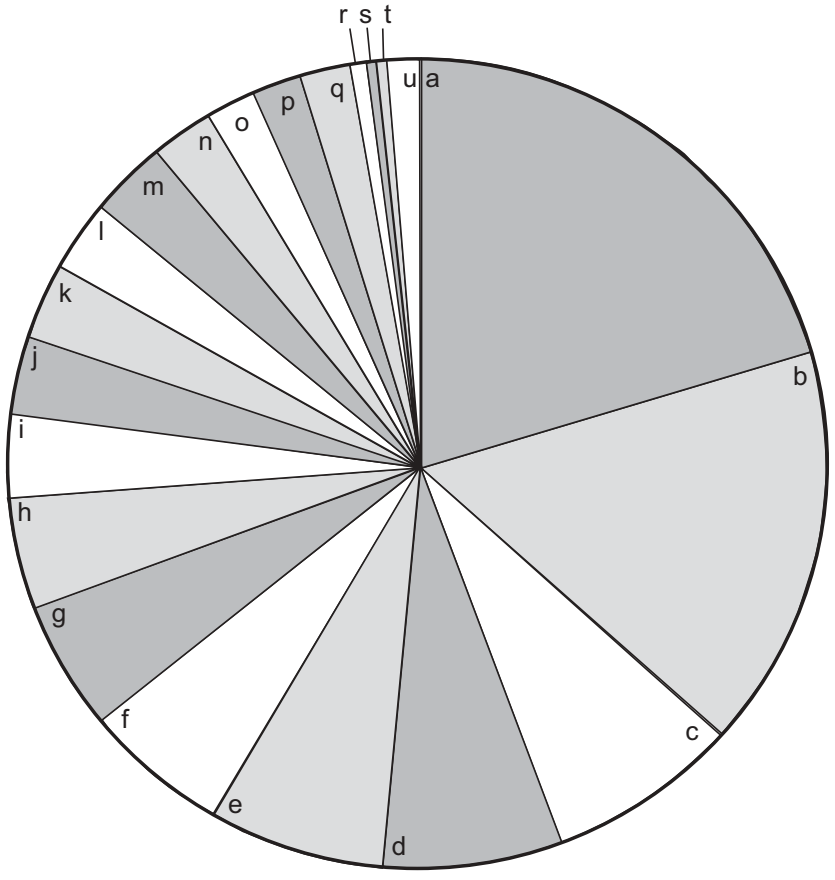

FIGURE 3. The most common differential diagnosis offered for case 3. a: psychiatric disease (20.7\%); b: asthma (15.9\%); c: cystic fibrosis (7.6\%); d: upper airways obstruction (7.3\%); e: bronchiectasis (7.0\%); f: bronchopulmonary aspergillosis (5.4\%); g: $\alpha_{1}$-antitrypsin deficiency $(5.4 \%) ; \mathrm{h}$ : chronic obstructive pulmonary disease (4.3\%); i: bronchopulmonary thromboembolic disease (3.3\%); j: cardiac (3.2\%); k: muscle weakness (3.0\%); I: diffuse parenchymal lung disease (2.9\%); m: Churg-Strauss syndrome (2.9\%); n: bronchiolitis (2.7\%); o: poor compliance (1.9\%); p: congenital problem (1.9\%); q: other (1.9\%), including the following differential diagnoses in decreasing order of importance, allergic disease with allergen/allergic component, other respiratory disease, or rare condition, drugrelated illness, ear, nose and throat and misdiagnosis of respiratory disorder; r: lung cancer $(0.6 \%)$; s: reflux oesophagitis $(0.4 \%)$; t: histiocytosis $(0.4 \%)$; u: miscellaneous $(1.3 \%)$, including the following differential diagnoses, which less than four respondents had suggested, obesity, chest wall deformity, sleep, systemic disorder, foreign body, occupational lung disease, pneumonia, Langerhans' cell histiocytosis.

\begin{tabular}{lc}
\hline TABLE 1 & $\begin{array}{l}\text { Most common misdiagnoses for difficult asthma } \\
\text { reported by respondents (Case 3) }\end{array}$ \\
Misdiagnoses & Subjects n \\
\hline Psychiatric disease & 212 \\
COPD & 182 \\
Vocal cord dysfunction & 111 \\
Bronchiectasis & 72 \\
Cardiac disease & 38 \\
Other mistaken diagnoses \# & 199 \\
$\quad$ Reflux oesophagitis & 29 \\
Poor compliance & 25 \\
Diffuse parenchymal lung disease & 22 \\
Tumours including lung cancer and carcinoid tumours & 18 \\
Churg-Strauss syndrome & 4 \\
Foreign bodies & 3 \\
Cystic fibrosis & 3 \\
\hline & \\
\hline & \#: the most common of which are detailed.
\end{tabular}

pulmonologists. It is possible that the survey results were not representative, but a response rate of $50 \%$ from busy secondary care respiratory professionals, in a country where the majority of patients with asthma are cared for in primary care, is probably respectable. Furthermore, the pattern of respiratory care in the UK is such that there is increasing sub-specialisation by respiratory specialists, with some spending the majority of time caring for those with lung cancer, and others mainly caring for those with sleep-related breathing disorders. It is thus possible that a large proportion of British respiratory physicians have only a minimal responsibility for the care of those with asthma and this may account for many not returning the questionnaire. This does not, however, diminish the value of this report; the authors have sub-analysed the results according to whether the respondents reported having a specialist responsibility and interest in the care of those with difficult asthma, or a more partial responsibility. Differences in

TABLE 2 Specialist versus nonspecialist care for difficult asthma

\begin{tabular}{|c|c|c|c|}
\hline & Specialists ${ }^{\#}$ & Nonspecialists" & p-value \\
\hline Subjects $\mathrm{n}$ & 21 & 152 & \\
\hline Prednisolone assays/cortisols & $5 / 20(25)$ & 17/127 (13.4) & 0.028 \\
\hline \multicolumn{4}{|l|}{ Skin-prick testing } \\
\hline \multicolumn{4}{|l|}{ Liaison psychiatrist } \\
\hline Easily available by referral & $15 / 21(71.4)$ & 47/142 (33.1) & $<0.001$ \\
\hline Difficult to access & 6/21 (28.6) & $94 / 142(66.1)$ & 0.001 \\
\hline Difficult asthma patient load & $173.09 \pm 232.7$ & $10.43 \pm 13.60^{* *}$ & $<0.001$ \\
\hline
\end{tabular}

Data are expressed as number of positive responses/number of respondents (\%) and mean \pm SD, unless otherwise stated. The word "specialist" here relates to whether the respondent reported themselves as having a special interest in the care of those with difficult asthma. " : reporting a special interest in difficult asthma; ": not the lead for asthma or difficult asthma; ${ }^{+}: p=0.002 ;{ }^{\S}: p=0.028 ;{ }^{f}: p=0.012 ;{ }^{* \star}: p \leqslant 0.001$. 


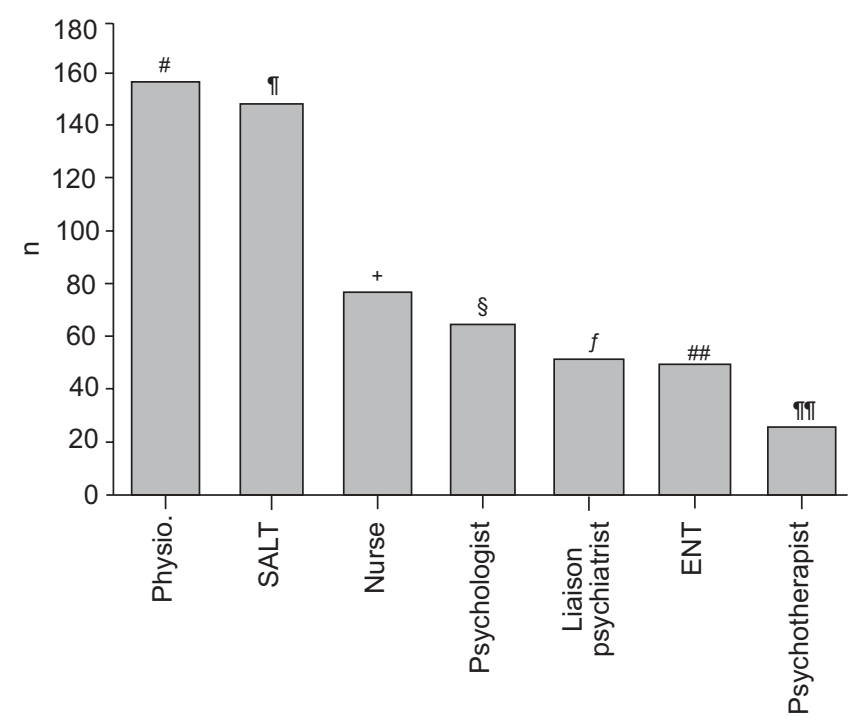

Healthcare professional

FIGURE 4. Healthcare professionals who are most involved with therapy of vocal cord dysfunction. Physio.: physiotherapist; SALT: speech and language therapy; ENT: ear, nose and throat. ${ }^{\#}$ : $27.5 \%$; ${ }^{\bullet}: 25.9 \%,{ }^{+}: 13.5 \% ;{ }^{\S}: 11.2 \%$; ${ }^{f}: 8.9 \%$; $\#$ \#:8.6\%;, : $4.4 \%$.

care were noted between these two groups and studies are needed elsewhere in Europe to determine whether the 1998 Task Force recommendations [1] are better implemented elsewhere.

This survey would suggest that if a patient with difficult asthma succeeds in seeing a respiratory specialist with a special interest in their condition, such a physician is more likely to have optimally configured their services. This is important when others $[5,6]$ have shown high rates of nonadherence with oral steroid therapy and significant psychiatric comorbidity in those with difficult asthma.

The 21 respondents in this study who reported that they had a special interest in difficult asthma were more likely to undertake allergy testing, assess compliance with drug assays and utilise psychiatric opinions. Respondents who did not express a special interest in difficult asthma report that access to liaison psychiatrists, psychologists and social workers was poor. It seems unlikely that the complex psychological problems sometimes suffered by these patients, and the risks resulting from such comorbidity [11] can be properly managed without good liaison psychiatry and psychology services. Those with a specialist interest in difficult asthma were also far more likely to routinely perform skin-prick test reactions for fungi than the generalists, and positive reactions to fungi have been reported to be more common amongst those with potentially fatal asthma [12], although whether the process of identifying hypersensitivity to fungi affects outcomes is unclear. Even amongst those who report a special interest in difficult asthma, it was only a minority who objectively tested patient compliance with medication.

In addition to the problems of noncompliance and psychological comorbidity, the wide differential diagnosis of difficult asthma was also highlighted by this survey. The list of diseases that were reported as masquerading as difficult asthma also emphasises the importance of differentiating localised airway obstruction from generalised obstruction. Some examples of misdiagnosis reported in this survey were of fixed structural localised obstructions, such as tumours or post-tracheostomy stenosis, but some were functional. The problem of vocal cord dysfunction (also known as glottic wheezing or functional upper airway obstruction) was well recognised by respondents and the condition is common. In one emergency department study, it was shown that $22 \%$ of patients attending with wheezing had vocal cord dysfunction [13]. However, there seems to be little consensus on the optimal method of confirming the diagnosis, if such confirmation is needed, and even less consensus on management. This probably reflects omission of the subject from guidelines as none of the major national or international guidelines include the subject of vocal cord dysfunction. It has been included in a recent European Respiratory Monograph [14], which considered a number of diagnostic tests and suggested that direct examination of vocal cords is diagnostic, revealing characteristic adduction of the vocal cords in association with wheezing. However, this may be present only intermittently. That particular issue of the European Respiratory Monograph [14] recognises that management consists of reassurance, which can be difficult, particularly when the condition co-exists with asthma. Psychiatric assessment is reported to be necessary and speech therapy useful [14].

The current survey cannot reveal the size of the problem of difficult asthma, but 7,000 patients with this diagnosis were reported as being under the care of British respiratory physicians; this is likely to be an underestimation. The full range of investigations and services necessary for these complex patients does not appear to be available in every respiratory service, and there may be advantages in these patients being referred to a specific "difficult asthma clinic". This should permit better characterisation of these patients, an enhanced understanding and a more rational approach to the evaluation of therapies and interventions. It can then be studied whether such optimisation of the process of care is associated with improved outcomes. The subject of difficult asthma also needs to be specifically addressed within national and international guidelines; this would demonstrate the importance of the subject and highlight gaps in the evidence, which should provide an added impetus for further research.

\section{REFERENCES}

1 Chung KF, Godard P, Adelroth E, et al. Difficult/therapyresistant asthma: the need for an integrated approach to define clinical phenotypes, evaluate risk factors, understand pathophysiology and find novel therapies. ERS Task Force on Difficult/Therapy-Resistant Asthma. Eur Respir J 1999; 13: 1198-1208.

2 The ENFUMOSA study group. The ENFUMOSA crosssectional European multicentre study of the clinical phenotype of chronic severe asthma. European Network for Understanding Mechanisms of Severe Asthma. Eur Respir J 2003; 22: 470-477.

3 Dolan CM, Fraher KE, Bleecker ER, et al. Design and baseline characteristics of the epidemiology and natural 
history of asthma: Outcomes and Treatment Regimens (TENOR) study: a large cohort of patients with severe or difficult-to-treat asthma. Ann Allergy Asthma Immunol 2004; 92: 32-39.

4 Irwin RS, Curley FJ, French CL. Difficult-to-control asthma. Contributing factors and outcome of a systematic management protocol. Chest 1993; 103: 1662-1669.

5 Robinson D, Campbell D, Durham S, Pfeffer J, Barnes P, Chung K. Systematic assessment of difficult to treat asthma. Eur Respir J 2003; 22: 478-483.

6 Heaney L, Conway E, Kelly C, et al. Predictors of therapy resistant asthma: outcome of a systematic evaluation protocol. Thorax 2003; 58: 561-566.

7 Lopez-Vina A, Aguero-Balbin R, Aller-Alvarez J, et al. Recommendations of the Spanish Society of Pulmonology and Thoracic Surgery (SEPAR). Guidelines for the Diagnosis and Management of Difficult to Control Asthma. Arch Bronchoneumol 2005; 41: 513-523.
8 Partridge MR, Latouche D, Trako E, Thurston JG. A national census of those attending UK accident and emergency departments with asthma. The UK National Asthma Task Force. J Accid Emerg Med 1997; 14: 16-20.

9 Van Ganse E, Laforest L, Pietri G, et al. Persistent asthma: disease control, resource utilisation and direct costs. Eur Respir J 2002; 20: 260-267.

10 Antonicelli L, Bucca C, Neri M, et al. Asthma severity and medical resource utilisation. Eur Respir J 2004; 23: 723-729.

11 Harrison BD. Difficult asthma. Thorax 2003; 58: 555-556.

12 Black PN, Udy AA, Brodie SM. Sensitivity to fungal allergens is a risk factor for life-threatening asthma. Allergy 2000; 55: 501-504.

13 Pearson M. Vocal-cord dysfunction often diagnosed as asthma. The Lancet 1999; 354: 1707.

14 Chung K, Sterling R, Chanez P, Godard P. Severe therapy resistant asthma. Eur Respir Mon 2003; 8: 312-338. 